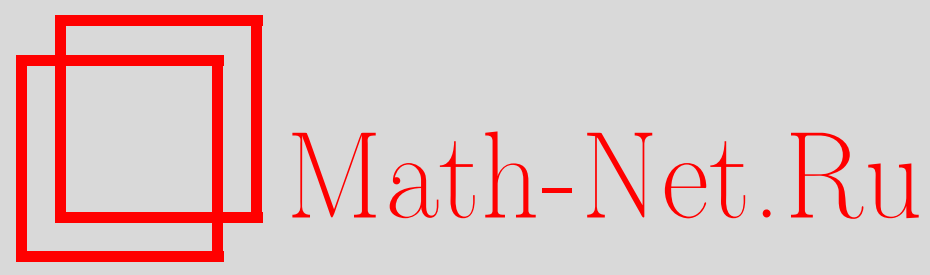

Ю. В. Муранов, Д. Реповш, Перестройки замкнутых многообразий с диэдральной фундаментальной группой, Матем. заметки, 1998, том 64, выпуск 2, 238-250

DOI: https://doi.org/10.4213/mzm1392

Использование Общероссийского математического портала Math-Net.Ru подразумевает, что вы прочитали и согласны с пользовательским соглашением http://www.mathnet.ru/rus/agreement

Параметры загрузки:

IP: 35.173 .219 .149

26 апреля 2023 г., $15: 16: 50$

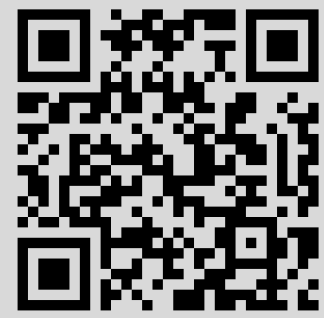




\title{
ПЕРЕСТРОЙКИ ЗАМКНУТЫХ МНОГООБРАЗИЙ С ДИЭДРАЛЬНОЙ ФУНДАМЕНТАЛЬНОЙ ГРУППОЙ
}

\author{
Ю.В. Муранов, Д. Реповш
}

В работе вычислены группы препятствий к перестройкам до простой гомотопической эквивалентности нормальных степени 1 отображений замкнутых многообразий $\mathrm{c}$ диэдральной фундаментальной группой. Рассмотрены случаи тривиальной ориентации диэдральной группы и нетривиальной ориентации на циклической подгруппе порядка 2. Получены новые резултаты о группах Браудера-Ливси и естественных отображениях $L$-групп, возникающих при вложении индекса 2 циклической группы в диэдральную.

Библиография: 16 названий.

1. Введение. Групшы Уолла $L_{n}^{s}(G, w)$ являются группами препятствий к перестройкам нормального степени 1 отображения $f:(M, \partial M) \rightarrow(Y, \partial Y)$ гладких (кусочно-линейных, топологических) многообразий с краем до простой гомотопической эквивалентности. Далее для этих групп будем использовать обозначение $L_{n}(G, w)$.

Здесь $n$ - размерность многообразия $Y, G=\pi_{1}(Y)$ и $w: G \rightarrow\{ \pm 1\}$ - гомоморфизм ориентации. Если край многообразия $Y$ непуст, то предполагаем, что ограничение отображения $f$ на край многообразия $M$ является простой гомотопической эквивалентностью $\left.f\right|_{\partial м}: \partial M \rightarrow \partial Y$. Каждый элемент групшы $L_{n}(G, w)$ реализуется нормальньпм отображением многообразий с краем (см. [1]). Однако, в случае конечной фундаментальной группы $G$ обычно сушествует очень мало элементов группы $L_{n}(G, w)$, которые могут быть реализованы нормальньми отображениями замкнутых многообразий. Подгруппу, порожденную такими элементами, обозначим через $C_{n}(G, w)$ (см. [2]).

Существует естественное отображение груп Уолла $L_{n}(G, w)$ в проективные группы Новикова-Уолла $L_{n}^{p}(G, w)$, которые устроены значительно проще. В работе [2] получен полный набор инвариантов, описьвающий образ групы $C_{n}(G, w)$ в групе $L{ }_{n}^{p}(G, w)$ для любой конечной 2-группы $G$. В случае тривиальной ориентации глубокие результаты по проблеме реализации получены в работе [3], а в случае нетривиальной ориентации полные результаты получены только для конечных абелевых 2-групп (см. [3], [4]). Техника, развитая Хэмблтоном в работе [2] для проективного случая, базируется на результатах Браудера, Ливси [5] и Кэппелла, Шейнсона [6]. Основную роль при этом играют группы Браудера-Ливси $L N_{n-1}(\pi \rightarrow G)$, где $\pi$ - подгруппа индекса 2 в группе $G$, и естественные отображения между ними и группами Уолла. Так, в работе [6] описано отображение, называемое инвариантом Браудера-Ливси, групшы $L_{n}(G)$ в группу

Работа первого автора выполнена при финансовой поддержке гранта Президента Российской Федерации, грант № 96-15-96841. Работа второго автора выполнена при подлержке Министерства науки и техники Республики Словения, грант № J1-7039-0101-95. 
Браудера-Ливси, которое тривиально на элементах группы Уолла, реализуемых нормальными отображениями замкнутых многообразий. Групшы Браудера-Ливси являются группами препятствий к расщеплению простой гомотопической эквивалентности $f: M \rightarrow Y$ вдоль одностороннего подмногообразия $X \subset Y$, когда индуцированное вложением отображение фундаментальных групп $\pi_{1}(X) \rightarrow \pi_{1}(Y)$ есть изоморфизм. Естественные отображения (трансфер, скрученный трансфер, индуцирование) между группами Уолла и группами Браудера-Ливси, необходимые для исследования проблемы реализации замкнутыми многообразиями, входят в косу Левина (см. [7], [8])

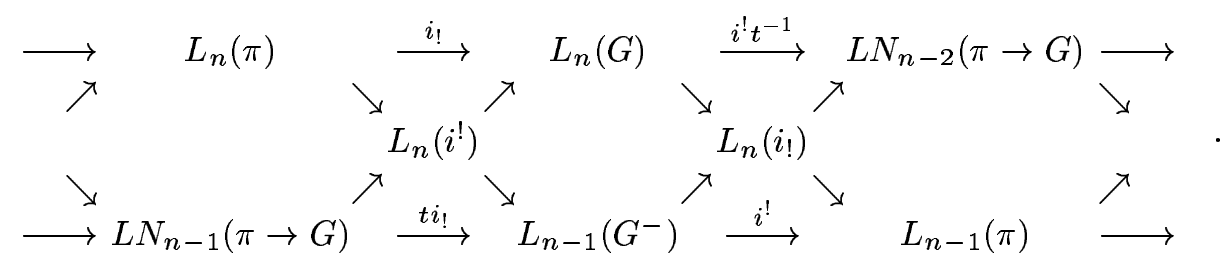

В геометрических приложениях наиболее важными оказываются отображения, входящие в верхнюю и нижнюю строки диаграммы (1) (см. [2], [6]). Диаграммы, в которых мы рассматриваем только верхнюю и нижнюю строки, будем называть двустрочными. Отметим, что верхняя и нижняя строки диаграммы (1) являются цепными комплексами с изоморфными в соответствующих членах гомологиями. Это обстоятельство дает достаточно эффективный метод вычисления групп и отображений. Знак “-” означает, что гомоморфизм ориентации изменен на образующей $t \in G \backslash \pi$. Отображение $i$ ! индуцировано вложением $i$, а отображение $i^{!}$есть трансфер в группах Уолла. Если $t-$ произвольный элемент из $G \backslash \pi$, то определен изоморфизм скейлинга групп Уолла для колец с антиструктурами (см. п. 2) $t: L_{n}(G, w) \rightarrow L_{n}\left(\mathbb{Z} G, \beta, w(t) t^{2}\right)[7],[8]$, где $\beta(x)=t \alpha(x) t^{-1}$. Отметим, в частности, что отображение $i^{!} t^{-1}$ в диаграмме (1) задает инвариант Браудера-Ливси. Существует также аналогичная (1) диаграмма, начинающаяся с отображения $i_{!}: L_{n}(\pi) \rightarrow L_{n}\left(G^{-}\right)$. Чтобы различать их, диаграмму (1) будем обозначать через $(D)$, а другую - через $(\widetilde{D})$.

Пусть $\pi=\mathbb{Z} / 2^{r} \subset D_{r+1}$ - вложение индекса 2 циклической групшы с тривиальной ориентацией в диэдральную 2-группу $D_{r+1}=\left\{x^{2^{r}}=y^{2}=1, y^{-1} x y=x^{-1}\right\}$. При $r=2$ группы $L_{n}\left(D_{r+1}, w\right)$ и $L_{n}^{p}\left(D_{r+1}, w\right)$ изоморфны при любом гомоморфизме ориентации $w$. Следовательно, результаты [2] дают полное описание груп $C_{n}\left(D_{3}, w\right)$ для любого $n=0,1,2,3 \bmod 4$. При $r \geqslant 3$ такого изоморфизма уже нет, значит в этом случае результаты [2] дают только нижнюю оценку групп $C_{n}\left(D_{r+1}, w\right)$.

В данной работе вычисляются групшы $C_{n}\left(D_{r+1}, w\right)$ для случаев, когда гомоморфизм ориентации $w$ тривиален на образующей $x$ групшы $D_{r+1}$. Групшы Уолла диэдральной группы в этих случаях вычислены в работе [8], а групшы Браудера-Ливси - в работе [9]. Основная трудность состоит в вычислении естественных отображений в диаграмме (1). В п. 3 для рассматриваемых вложений вычислены отображения в диаграмме (1), а также группа $L N_{1}\left(\pi \rightarrow D_{r+1}^{+,+}\right) \cong L N_{3}\left(\pi \rightarrow D_{r+1}^{+,-}\right)$, полученная в работе [9], с точностью до расширения. Для вычислений использована спектральная последовательность в теории перестроек из работы [10]. Отметим, что в случае тривиальной ориентации на группе $D_{r+1}(w(y)=w(x)=1)$ группы $C_{n}\left(D_{r+1}, w\right)$ можно также вычислить (см. [3]). Основными результатами работы являются теоремы $3-5$.

Промежуточньй шаг в вычислении групп препятствий к перестройкам и груп Браудера-Ливси (см. [8]) состоит в вычислении групा $L_{*}^{Y}\left(\widehat{\mathbb{Z}}_{2} \pi\right)$, где $\widehat{\mathbb{Z}}_{2}-$ кольцо целых 
2 -адических чисел, $Y=\operatorname{ker}\left(K_{1}(\mathbb{Z} \pi) \rightarrow K_{1}(\mathbb{Q} \pi)\right) \cup\{ \pm \pi\}$. Эти группы входят в точную последовательность Ротенберга (см. [8])

$$
\longrightarrow L_{n}^{Y}\left(\widehat{\mathbb{Z}}_{2} \pi\right) \longrightarrow L_{n}^{K}\left(\widehat{\mathbb{Z}}_{2} \pi\right) \longrightarrow H^{n}(K / Y) \longrightarrow
$$

где $H^{n}(K / Y)$ - когомологии Тейта группы $K / Y, K=K_{1}\left(\widehat{\mathbb{Z}}_{2} \pi\right)$, и инволюция на группе $K / Y$ индуцирована стандартной инволюцией на групповом кольце $\widehat{\mathbb{Z}}_{2} \pi$, заданной формулой

$$
\Sigma n_{g} g \rightarrow \Sigma n_{g} w(g) g^{-1}, \quad n_{g} \in \widehat{\mathbb{Z}}_{2}, \quad g \in \pi,
$$

и $w: \pi \rightarrow\{ \pm 1\}$ - гомоморфизм ориентации. Для конечной 2 -группы $\pi$ групша $L_{*}^{K}\left(\widehat{\mathbb{Z}}_{2} \pi\right)$ изоморфна $\mathbb{Z} / 2$. Группы $L_{*}^{Y}\left(\widehat{\mathbb{Z}}_{2} \pi\right)$ и аналогичные грушы для антиструктур, соответствующих группам Браудера-Ливси, вычислены в работах [8], [9]. Естественные отображения между этими группами можно вычислить, используя естественные отображения когомологий Тейта (см. [11]-[13]).

Для любой групшы $\pi$ имеет место относительная длинная точная последовательность (cм. [8])

$$
\longrightarrow L_{n}^{\prime}(\mathbb{Z} \pi) \longrightarrow L_{n}^{\prime}\left(\widehat{\mathbb{Z}}_{2} \pi\right) \longrightarrow L_{n}\left(\mathbb{Z} \pi \rightarrow \widehat{\mathbb{Z}}_{2} \pi\right) \longrightarrow ，
$$

в которой $L_{n}^{\prime}\left(\widehat{\mathbb{Z}}_{2} \pi\right)$ является группой $L_{n}^{Y}\left(\widehat{\mathbb{Z}}_{2} \pi\right)$ при $n=2 k$ и отождествляется с ядром отображения $L_{n}^{Y}\left(\widehat{\mathbb{Z}}_{2} \pi\right) \rightarrow L_{n}^{K}\left(\widehat{\mathbb{Z}}_{2} \pi\right)$ при $n=2 k+1$. Отметим, что для диэдральной либо циклической 2 -группы $\pi$ группа

$$
S K_{1}(\mathbb{Z} \pi)=\operatorname{ker}\left(K_{1}(\mathbb{Z} \pi) \rightarrow K_{1}(\mathbb{Q} \pi)\right)
$$

тривиальна (см. [8]). Следовательно, в рассматриваемом случае имеет место изоморфизм $L^{\prime} \cong L^{s}$. В дальнейшем все $L^{s}$-группы будем обозначать через $L$.

Таким образом, для вычисления естественных отображений в диаграмме (1) для вложения $i: \pi \rightarrow G$ индекса 2 можно использовать двустрочные диаграммы для квадратичного расширения $\widehat{\mathbb{Z}}_{2} \pi \rightarrow \widehat{\mathbb{Z}}_{2} G$ (см. [7]) и двустрочные диаграммы для относительных групп (см. [11]).

2. Естественные отображения когомологий Тейта. Этот пункт содержит необходимые предварительные сведения о группах Уолла для колец с антиструктурами и спектральных последовательностях в теории перестроек для квадратичных расширений антиструктур (см. [7]-[14]).

Антиструктура - это тройка $(R, \alpha, u)$, где $R$ - кольцо с единицей, $u \in R^{*}$ - обратимьй элемент кольца, $\alpha: R \rightarrow R$ - антиавтоморфизм кольца $R$ такой, что $\alpha(u)=u^{-1}$, $\alpha^{2}(x)=u x u^{-1}$ для любого $x \in R$. Cтруктура на кольце $R$ - это пара $(\rho, a)$, где $\rho: R \rightarrow R$ - автоморфизм кольца $R$, а $a$ - обратимый элемент такой, что $\rho(a)=a$, $\rho^{2}(x)=a x a^{-1}$ для любого $x \in R$ (см. [7]). Пусть $S=R[t] /\left(t^{2}-a\right)$ - квадратичное расширение кольца $R$, где $t$ - элемент независимый над $R$, для которого $\rho(x)=t x t^{-1}$ для любого $x \in R$. Пусть также сушествует продолжение автоморфизма $\alpha$ на кольцо $S$ такое, что $\alpha(t) t \in R \subset S, \alpha^{2}(t)=u t u^{-1} \in S$. В этом случае антиструктура $(S, \alpha, u)$ назьвается квадратичным расширением антиструктуры $(R, \alpha, u)$. Автоморфизм $\rho$ продолжается на кольцо $S$ по формуле $\rho(x+y t)=t(x+y t) t^{-1}, x, y \in R$. Можно также определить автоморфизм $\gamma$ кольца $S$, полагая $\gamma(x+y t)=(x-y t), x, y \in R$. По данному квадратичному расширению антиструктур можно построить другое квадратичное расширение $(R, \widetilde{\alpha}, \widetilde{u}) \rightarrow(S, \widetilde{\alpha}, \widetilde{u})$, где $\widetilde{\alpha}=\rho \gamma \alpha$ и $\widetilde{u}=-t \alpha\left(t^{-1}\right) u($ см. [7]). Так как квадраты 
антиавтоморфизмов $\alpha, \widetilde{\alpha}$ являются внутренними автоморфизмами, то эти антиавтоморфизмы индуцируют инволюции на группах $K_{j}(R), K_{j}(S)$ для $j=0,1$. Обозначим эти инволюции через $T$ и $\widetilde{T}$ соответственно.

Для любой подгруппы $X \subset K_{j}(R)$, инвариантной относительно инволюции $T$, определены групшы Уолла с декорациями $L_{n}^{X}(R, \alpha, u)$, так что для двух инвариантных подгрупп $X \subset X^{\prime} \subset K_{j}(R)$ имеет место точная последовательность Ротенберга (см., например, [8], [14])

$$
\longrightarrow L_{n}^{X}(R) \longrightarrow L_{n}^{X^{\prime}}(R) \longrightarrow H^{n}\left(X^{\prime} / X\right) \longrightarrow
$$

где $H^{*}$ - когомологии Тейта. Пусть $i:(R, \alpha, u) \rightarrow(S, \alpha, u)$ - квадратичное расширение антиструктур. Тогда для $j=0,1$ определен гомоморфизм индуцирования $i_{*}: K_{j}(R) \rightarrow$ $K_{j}(S)$ и гомоморфизм переноса $i^{*}: K_{j}(S) \rightarrow K_{j}(R)$. Отображения $i^{*}, i_{*}$ коммутируют с инволюциями $T, \widetilde{T}$ и, следовательно, индуцируют отображения когомологий Тейта,

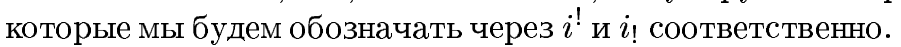

Аналогично, пусть $X \subset X^{\prime} \subset K_{j}(R)$ и $Y \subset Y^{\prime} \subset K_{j}(S)$ являются $T$-, $\widetilde{T}$-инвариантными подгруппами при $j=0,1$. Предположим, что $i_{*}(X) \subset Y, i^{*}(Y) \subset X, i_{*}\left(X^{\prime}\right) \subset Y^{\prime}$, $i^{*}\left(Y^{\prime}\right) \subset X^{\prime}$, и обозначим $A=X^{\prime} / X, B=Y^{\prime} / Y$. В этом случае мы также получаем индуцированные $i^{*}, i_{*}$ отображения когомологий Тейта групп $A$ и $B$, которые,

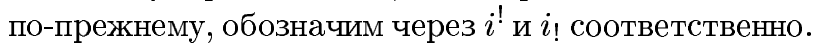

В этих предположениях существует коса Левина, состоящая из относительных точных последовательностей когомологий Тейта (см. [11], [12]):

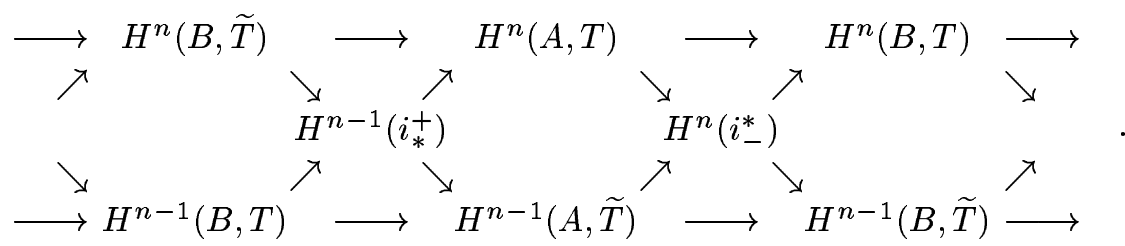

В эту диаграмму входят относительные точные последовательности для отображений индуцирования

$$
i_{*}:(A, T) \rightarrow(B, T), \quad i_{*}:(A, \widetilde{T}) \rightarrow(B, \widetilde{T})
$$

и относительные точные последовательности для отображений переноса

$$
i^{*}:(B, T) \rightarrow(A, \widetilde{T}), \quad i^{*}:(B, \widetilde{T}) \rightarrow(A, T) .
$$

Обозначим эту диаграмму через $(D)$. Другая диаграмма $(\widetilde{D})$ получается для отображений индуцирования

$$
i_{*}:(A, \widetilde{T}) \rightarrow(B, T), \quad i_{*}:(A, T) \rightarrow(B, \widetilde{T})
$$

и отображений переноса

$$
i^{*}:(B, T) \rightarrow(A, T), \quad i^{*}:(B, \widetilde{T}) \rightarrow(A, \widetilde{T})
$$

Диаграмма (1) реализуется на уровне спектров (см. [10]). С использованием этого факта в [10] построена спектральная последовательность в теории перестроек, причем $E_{1}^{p, q}=L N_{q+2}(\pi \rightarrow G)$, а первый дифференциал совпадает с композицией

$$
L N_{n}\left(\pi \rightarrow G^{ \pm}\right) \longrightarrow L_{n}\left(G^{\mp}\right) \longrightarrow L N_{n-2}\left(G^{\mp}\right)
$$


где одно отображение из диаграммы $(D)$, а другое отображение из диаграммы $(\widetilde{D})$. При этом первый дифференциал совпадает с отображением $1 \mp \Phi$, где $\Phi$ - инволюция на группах Браудера-Ливси (см. [2], [10]). Диаграмма (4) реализуется на уровне спектров (см. [11], [13]) аналогично диаграмме (1). Таким образом, метод Хэмблтона и Харшиладзе [10], построивших спектральную последовательность в теории перестроек по диаграмме (1), дает спектральную последовательность когомологий Тейта в рассматриваемом случае. Подробное построение спектральной последовательности в этом случае содержится в работе [13]. Первьй дифференциал $d_{1}^{p, q}$ для четного $p$ и $q=0,1$ совпадает с композицией

$$
H^{q}(A, \widetilde{T}) \stackrel{i_{!}}{\longrightarrow} H^{q}(B, \widetilde{T}) \stackrel{i_{!}}{\longrightarrow} H^{q}(A, \widetilde{T})
$$

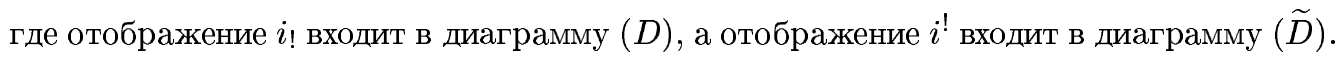
Для нечетного $p$ и $q=0,1$ первьй дифференциал $d_{1}^{p, q}$ совпадает с композицией

$$
H^{q}(A, \widetilde{T}) \stackrel{i_{!}}{\longrightarrow} H^{q}(B, T) \stackrel{i_{!}}{\longrightarrow} H^{q}(A, \widetilde{T})
$$

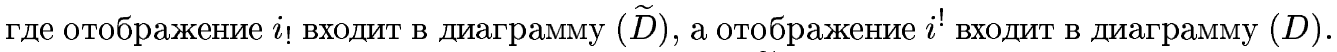
Обозначим через $\rho$ инволюцию на групе $H^{q}(A, \widetilde{T})$, индуцированную инволюцией $\rho$ на кольце $R$.

Теорема 1 [13]. Дифференциал $d_{1}^{p, q}: E_{1}^{p, q} \rightarrow E_{1}^{p+1, q}$ не зависит от $p$ и $q$ и совпадает с гомоморфизмом $1+\rho$.

Пусть $\pi=\mathbb{Z} / 2^{r} \subset D_{r+1}=G$ - вложение индекса 2 циклической групшы с тривиальной ориентацией в диэдральную группу с тривиальной ориентацией, $w=1$. Через $i^{-}$ будем обозначать вложение $\pi \rightarrow G^{-}$групп с ориентацией. Если из контекста ясно, какую ориентацию мы рассматриваем, то не будем указывать ориентацию в обозначениях групп и отображений. Вложение $i$ индуцирует квадратичное расширение колец со стандартной инволюцией $\widehat{\mathbb{Z}}_{2} \pi \rightarrow \widehat{\mathbb{Z}}_{2} G$. Инволюция $\rho$ в этом случае задается на любом элементе $x \in \pi$ равенством $\rho(x)=x^{-1}$. Обозначим $A=K_{1}\left(\widehat{\mathbb{Z}}_{2} \pi / Y\right), B=K_{1}\left(\widehat{\mathbb{Z}}_{2} G / Y\right)$. Тогда $H^{1}(A, T)=H^{1}(A, \widetilde{T})=H^{1}(B, T)=H^{1}(B, \widetilde{T})=0, H^{0}(A, T)=(\mathbb{Z} / 2)^{2}, H^{0}(A, \widetilde{T})=$ $(\mathbb{Z} / 2)^{2^{r}}, H^{0}(B, T)=(\mathbb{Z} / 2)^{2^{r-1}+3}, H^{0}(B, \widetilde{T})=(\mathbb{Z} / 2)^{2^{r-1}-1}$ (cм. [8], [9]).

Теорема 2 [11], [13]. 1) В двустрочной диаграмме когомологий Тейта для вложения $i$ нетривиальна только четномерная строка, являющаяся точной последовательностью

$$
\longrightarrow H^{0}(A, T) \stackrel{i_{!}}{\longrightarrow} H^{0}(B, T) \stackrel{i^{!}}{\longrightarrow} H^{0}(A, \widetilde{T}) \stackrel{i_{!}}{\longrightarrow} H^{0}(B, \widetilde{T}) \stackrel{i^{!}}{\longrightarrow},
$$

в которой левое отображение является мономорфизмом.

2) В двустрочной диаграмме для вложения $i^{-}$нетривиальна только четномерная строка, являющаяся точной последовательностью

$$
\longrightarrow H^{0}(A, T) \stackrel{i_{!}}{\longrightarrow} H^{0}(B, \widetilde{T}) \stackrel{i^{!}}{\longrightarrow} H^{0}(A, \widetilde{T}) \stackrel{i_{!}}{\longrightarrow} H^{0}(B, T) \stackrel{i^{!}}{\longrightarrow}
$$

в которой левое отображение тривиально. 
3. Естественные отображения $L$-групп. В этом пункте вычислены естественные отображения в диаграмме (1) для вложения $i: \pi=\mathbb{Z} / 2^{r} \subset D_{r+1}=\left\{x^{2^{r}}=y^{2}=1\right.$, $\left.y^{-1} x y=x^{-1}\right\}=G$ и некоторые группы Браудера-Ливси.

Напомним, что мы рассматриваем только случай тривиальной ориентации на циклической подгруппе. Введем следующие обозначения: $R=\mathbb{Z} \pi, T^{ \pm}=\mathbb{Z} G^{ \pm}, \widehat{R}_{2}=\widehat{\mathbb{Z}}_{2} \pi$, $\widehat{T}_{2}^{ \pm}=\widehat{\mathbb{Z}}_{2} G^{ \pm}, A=K_{1}\left(\widehat{\mathbb{Z}}_{2} \pi\right) / Y, B=K_{1}\left(\widehat{\mathbb{Z}}_{2} G\right) / Y$. Знак "+" означает, что гомоморфизм ориентации $w$ тривиален на образующей $y$, а знак “-"означает, что $w(y)=-1$. В случае, когда мы рассматриваем когомологии Тейта относительно инволюции, индуцированной стандартной инволюцией в групповом кольце (она соответствует инволюции $T$ п. 2), будем писать $H^{n}(A)$ и $H^{n}(B)$. Когомологии Тейта, рассматриваемые относительно тождественной инволюции, соответствующей антиструктуре групп Браудера-Ливси, будем обозначать через $H^{n}(A, *)$ и $H^{n}(B, *)$ (она соответствует инволюции $\widetilde{T}$ п. 2). Напомним, что антиструктура $L_{n}\left(\widehat{\mathbb{Z}}_{2} \pi, \mathrm{Id}, 1\right)$ соответствует антиструктуре для групп Браудера-Ливси в рассматриваемом случае в силу изоморфизма $L N_{n+2}(\pi \rightarrow$ $\left.D_{r+1}^{+}\right) \cong L N_{n}\left(\pi \rightarrow D_{r+1}^{-}\right) \cong L_{n}(\mathbb{Z} \pi, \mathrm{Id}, 1)$.

Все групшы, входящие в диаграммы типа (1) для квадратичных расширений $\widehat{\mathbb{Z}}_{2} \pi \rightarrow$ $\widehat{\mathbb{Z}}_{2} G^{+}$и $\widehat{\mathbb{Z}}_{2} \pi \rightarrow \widehat{\mathbb{Z}}_{2} G^{-}$, известны из работ [8], [9]. Имеют место следующие изоморфизмы:

$$
\begin{aligned}
& L_{n}\left(\widehat{R}_{2}\right) \cong \begin{cases}0 & \text { при } n=0 \bmod 4, \\
(\mathbb{Z} / 2)^{2} & \text { при } n=1 \bmod 4, \\
\mathbb{Z} / 2 & \text { при } n=2,3 \bmod 4,\end{cases} \\
& L_{n}\left(\widehat{T}_{2}^{-}\right) \cong \begin{cases}\mathbb{Z} / 2 & \text { при } n=0,2 \bmod 4, \\
(\mathbb{Z} / 2)^{2^{r-1}-1} & \text { при } n=1,3 \bmod 4,\end{cases} \\
& L_{n}\left(\widehat{T}_{2}^{+}\right) \cong \begin{cases}0 & \text { при } n=0 \bmod 4, \\
(\mathbb{Z} / 2)^{2^{r-1}+3} & \text { при } n=1 \bmod 4, \\
\mathbb{Z} / 2 & \text { при } n=2 \bmod 4, \\
(\mathbb{Z} / 2)^{2^{r-1}+2} & \text { при } n=3 \bmod 4,\end{cases} \\
& L_{n}\left(\widehat{\mathbb{Z}}_{2} \pi, \operatorname{Id}, 1\right) \cong \begin{cases}0 & \text { при } n=0 \bmod 4, \\
(\mathbb{Z} / 2)^{2^{r}} & \text { при } n=1 \bmod 4, \\
\mathbb{Z} / 2 & \text { при } n=2 \bmod 4, \\
(\mathbb{Z} / 2)^{2^{r}-1} & \text { при } n=3 \bmod 4 .\end{cases}
\end{aligned}
$$

Лемма 1. 1) Отобрахение $i_{!}: L_{n}\left(\widehat{R}_{2}\right) \rightarrow L_{n}\left(\widehat{T}_{2}^{+}\right)$, индуцированное отображением $i$, является мономорфизмом при любом $n=0,1,2,3 \bmod 4$.

2) Отображсние $i_{!}: L_{n}\left(\widehat{R}_{2}\right) \rightarrow L_{n}\left(\widehat{T}_{2}^{-}\right)$является мономорфизмом при четном $n$ и тривиально при нечетном $n$, а отображсене $i^{!}: L_{2 n+1}\left(\widehat{T}_{2}^{+}\right) \rightarrow L_{2 n+1}\left(\widehat{R}_{2}\right)$ является эпиморфизмом при $n=0,1 \bmod 2$.

ДокАЗАТЕЛьСтво. В четных размерностях утверждения леммы либо тривиальны, либо следуют из сохранения Arf-инварианта отображением $i_{!}$. Последнее, в свою очередь, следует из очевидных изоморфизмов $L_{n}^{K}\left(\widehat{R}_{2}\right) \stackrel{\cong}{\longrightarrow} L_{n}^{K}\left(\widehat{T}_{2}^{ \pm}\right) \cong \mathbb{Z} / 2$. 
Рассмотрим коммутативную диаграмму

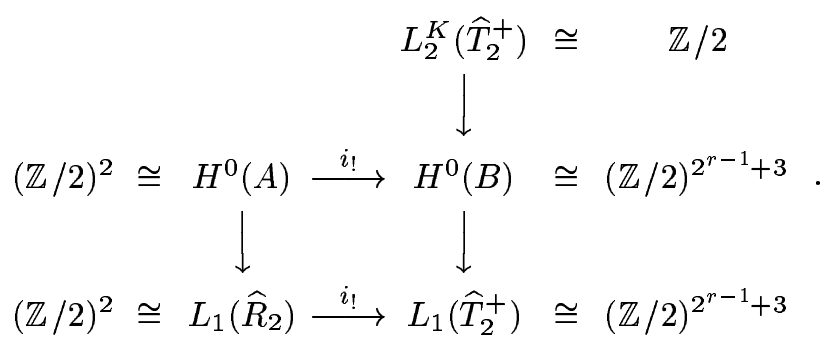

Правая вертикальная строка является точной последовательностью Ротенберга (2). Верхнее вертикальное отображение тривиально, так как в групе $L_{2}\left(\widehat{T}_{2}^{+}\right)$Arf-инвариант реализуется. Верхнее горизонтальное отображение является мономорфизмом по теореме 2. Следовательно, нижнее горизонтальное отображение также является мономорфизмом. В размерности 3 для случая 1) рассуждения аналогичны. В случае 2) рассмотрим коммутативную диаграмму

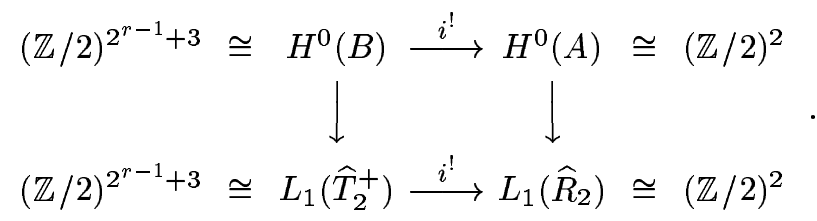

Верхнее горизонтальное отображение является эпиморфизмом по теореме 2. Правое вертикальное отображение является изоморфизмом, как следует из рассмотрения предыдущей диаграммы. Отсюда следует утверждение леммы в размерности 1. Размерность 3 рассматривается аналогично. Заметим также, что отображение $L_{2}\left(\widehat{\mathbb{Z}}_{2} \pi, \mathrm{Id}, 1\right) \rightarrow$ $L_{2}\left(\widehat{T}_{2}^{+}\right)$, входящее в одну из рассматриваемых диаграмм, является изоморфизмом. Оно сохраняет Arf-инвариант. Теперь остальные утверждения леммы получаются с помощью диаграммного поиска в двустрочных диаграммах. Лемма доказана.

Следует отметить, что полученные в лемме 1 результаты дают все отображения в диаграммах типа (1) для квадратичных расширений $\widehat{R}_{2} \rightarrow \widehat{T}_{2}^{ \pm}$.

Относительные грушы Уолла из точной последовательности (3) также известны (см. [8], [9]). Имеют место следуюшие изоморфизмы:

$$
\begin{aligned}
& L_{n}\left(R \rightarrow \widehat{R}_{2}\right) \cong \begin{cases}0 & \text { при } n=0,2 \bmod 4, \\
\mathbb{Z}^{2^{r-1}+1} \oplus(\mathbb{Z} / 2)^{2} & \text { при } n=1 \bmod 4, \\
\mathbb{Z}^{2^{r-1}-1} & \text { при } n=3 \bmod 4,\end{cases} \\
& L_{n}\left(T^{+} \rightarrow \widehat{T}_{2}^{+}\right) \cong \begin{cases}0 & \text { при } n=0,2,3 \bmod 4, \\
\mathbb{Z}^{2^{r-1}+3} \oplus(\mathbb{Z} / 2)^{r+3} & \text { при } n=1 \bmod 4,\end{cases} \\
& L_{n}\left(T^{-} \rightarrow \widehat{T}_{2}^{-}\right) \cong \begin{cases}0 & \text { при } n=0,1,2 \bmod 4, \\
(\mathbb{Z} / 2)^{r-1} \oplus \mathbb{Z}^{2^{r-1}-1} & \text { при } n=3 \bmod 4,\end{cases} \\
& L_{n}\left(R \rightarrow \widehat{R}_{2}, \mathrm{Id}, 1\right) \cong \begin{cases}(\mathbb{Z} / 2)^{r-1} & \text { при } n=0 \bmod 4, \\
\mathbb{Z}^{2} \oplus(\mathbb{Z} / 2)^{2^{r-1}+r} & \text { при } n=1 \bmod 4, \\
0 & \text { при } n=2,3 \bmod 4 .\end{cases}
\end{aligned}
$$

В диаграммах следующей леммы написаны только ненулевые относительные группы верхней и нижней строки. 
Лемма 2. 1) В двустрочной диаграмме $(D)$ относительных групп

$$
\begin{aligned}
& L_{1}\left(R \rightarrow \widehat{R}_{2}\right) \stackrel{i_{!}}{\longrightarrow} L_{1}\left(T^{+} \rightarrow \widehat{T}_{2}^{+}\right) \stackrel{i^{!} t^{-1}}{\longrightarrow} L_{1}\left(R \rightarrow \widehat{R}_{2}, \mathrm{Id}, 1\right) \stackrel{t i_{!}}{\longrightarrow} L_{3}\left(T^{-} \rightarrow \widehat{T}_{2}^{-}\right) \\
& \stackrel{i^{!}}{\longrightarrow} \quad L_{3}\left(R \rightarrow \widehat{R}_{2}\right) \\
& L_{0}\left(R \rightarrow \widehat{R}_{2}, \mathrm{Id}, 1\right)
\end{aligned}
$$

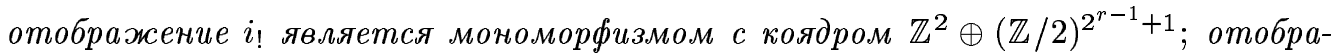
жение $i^{!} t^{-1}$ имеет коядро $(\mathbb{Z} / 2)^{r-1}$, лежащее в подгруппе кручения; отображение $t i_{\text {! }}$ является әпиморфизмом на подгруппу кручения; отображение $i^{!}$имеет коядро $(\mathbb{Z} / 2)^{r-1}$.

2) В двустрочной диаграмме $(\widetilde{D})$ относительных групп

$$
\begin{aligned}
L_{3}\left(R \rightarrow \widehat{R}_{2}\right) \stackrel{i_{!}}{\longrightarrow} L_{1}\left(T^{-} \rightarrow \widehat{T}_{2}^{-}\right) \stackrel{i^{\prime} t^{-1}}{\longrightarrow} L_{1}\left(R \rightarrow \widehat{R}_{2}, \mathrm{Id}, 1\right) \stackrel{t i_{!}}{\longrightarrow} \quad L_{1}\left(T^{+} \rightarrow \widehat{T}_{2}^{+}\right) \\
\stackrel{i^{!}}{\longrightarrow} \quad L_{1}\left(R \rightarrow \widehat{R}_{2}\right) \\
{ } \\
L_{0}\left(R \rightarrow \widehat{R}_{2}, \mathrm{Id}, 1\right)
\end{aligned}
$$

отображсение $i_{\text {! }}$ имеет коядро $(\mathbb{Z} / 2)^{2^{r-1}-1} ;$ отображсение $i^{!} t^{-1}$ имеет образ $(\mathbb{Z} / 2)^{2^{r-1}-1}$, лежсащий в подгруппе кручения; отображение $t i_{1}$ имеет образ $(\mathbb{Z} / 2)^{r+1} \oplus \mathbb{Z}^{2}$, являюшийся прямым слагаемым; отображение $i^{!}$является әпиморфизмом на подгруппу кручения и имеет коядро $(\mathbb{Z} / 2)^{r-1}$.

ДоказАтельство. Рассмотрим случай 1). Согласно [15] (см. также [11]) для любой конечной 2-групшы $\pi$ група $L_{*}\left(\mathbb{Z} \pi \rightarrow \widehat{\mathbb{Z}}_{2} \pi\right)$ изоморфна группе $L_{*}^{S}\left(\mathbb{Z}[1 / 2] \pi \rightarrow \widehat{\mathbb{Q}}_{2} \pi\right)$, где $S=0 \subset K_{1}$. Групша $L^{S}$ разлагается в прямую сумму в соответствии с разложением кольца $\mathbb{Z}[1 / 2] \pi$, и соответственно можно разложить в прямую сумму индуцированное $i$ отображение групповых колец (см. [11], [15]). В рассматриваемом случае двустрочная диаграмма относительных групп разлагается в прямую сумму

а) двух двустрочных диаграмм, соответствующих диагональному вложению

$$
\mathbb{Z}[1 / 2] \rightarrow \mathbb{Z}[1 / 2] \oplus \mathbb{Z}[1 / 2],
$$

б) $r-1$ двустрочных диаграмм, соответствующих квадратичньм расширениям

$$
\left(\Gamma_{k}, c, 1\right) \rightarrow M_{2}\left(R_{k-1}, \mathrm{Id}, 1\right), \quad k=1,2, \ldots, r-1
$$

(см. [11]), где $\Gamma_{k}=\mathbb{Z}[1 / 2]\left[\theta_{k+1}\right], R_{k+1}=\mathbb{Z}[1 / 2]\left[\theta_{k+2}+\bar{\theta}_{k+2}\right], c-$ комплексное сопряжение, $\theta_{m}$ - примитивньй корень степени $2^{m}$ из 1.

Диаграммы относительных групп для отображений из б) изоморфны двустрочньм диаграммам для вложений $\left(R_{k-1}, \mathrm{Id}, 1\right) \rightarrow\left(\Gamma_{k}, c, 1\right)$ с точностью до переобозначения отображений (см. [15]). Таким образом, все необходимые диаграммы из а) и б) описаны в работе [11] (это диаграмма $(D 4)$ при $N=1$ и диаграмма $(D 3)$ при $N=k$ ). Непосредственньй подсчет доказывает утверждение 1) леммы 2. Случай 2) рассматривается аналогично. Лемма доказана. 
Лемма 3. Пусть $A$ - конечная абелева 2-группа $и \Phi-$ инволюиия на группе $A$ такая, что гомоморфизмы $d^{ \pm}: A \rightarrow A$, заданные формулами $d^{+}(x)=x \Phi(x)$, $d^{-}(x)=x(\Phi(x))^{-1}$, имеют образы, являюшиеся әлементарными 2-группами и прямыми слагаемыми в группе $A$. Тогда $A$ - әлементарная 2-группа.

ДокАЗАтЕльство. Для любого элемента $x \in A$ рассмотрим подгруппу $\rho \subset A$, порожденную элементами $x$ и $\Phi(x)$. Если $\rho$ - циклическая подгруппа, то $x$ и $\Phi(x)$ являются ее образующими, так как $\Phi$ - инволюция. Если при этом $\rho$ имеет порядок 2 , то $x$ имеет порядок 2. Если $\rho$ имеет порядок больше 2 , то $x, \Phi(x) \in \operatorname{Im}\left(\left.\Phi\right|_{\rho}\right)$. Следовательно, $x \Phi(x)=x(\Phi(x))^{-1}=1$, так как единственная элементарная 2-группа, являющаяся прямым слагаемым в группе $\rho$, есть тривиальная группа. Таким образом, $(\Phi(x))^{2}=1$, a это противоречит предположению, что порядок $\rho$ больше 2 . Если $\rho$ не является циклической группой, то ее можно записать в виде $\rho_{1} \oplus \rho_{2}$, где $\rho_{1}$ и $\rho_{2}-$ циклические 2-групш, переставляемые инволюцией. Эти групш имеют образующие $x$ и $\Phi(x)$ соответственно. Следовательно, порядок элемента $x \Phi(x)$ равен порядку группы $\rho_{1}$, совпадающему с порядком группы $\rho_{2}$. По условию порядок элемента $x \Phi(x)$ равен 2 , т.е. групшы $\rho_{1}, \rho_{2}$ и элемент $x$ имеют порядок 2 . Лемма доказана.

Теорема 3. Имеет место изоморфизм

$$
L N_{3}\left(\mathbb{Z} / 2^{r} \rightarrow D_{r+1}^{+,-}\right) \cong L N_{1}\left(\mathbb{Z} / 2^{r} \rightarrow D_{r+1}^{+,+}\right) \cong(\mathbb{Z} / 2)^{2^{r}+r-2}
$$

ДокАЗАТЕЛЬСТво. С точностью до расширения группа $L N_{3}=L N_{3}\left(\mathbb{Z} / 2^{r} \rightarrow D_{r+1}^{+,-}\right)$ описана в работе [9]. Поэтому нам достаточно доказать, что эта групша элементарна. Рассмотрим коммутативную диаграмму

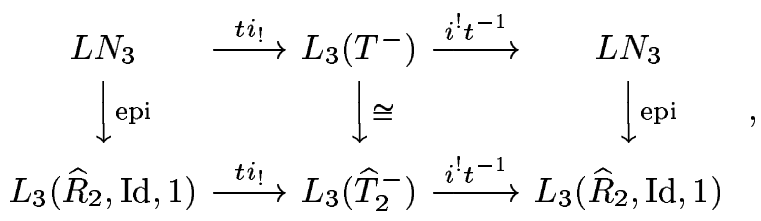

в которой крайние вертикальные отображения лежат в относительной точной последовательности (3) и являются эпиморфизмами согласно [9]. Левоенижнеегоризонтальное отображение является эпиморфизмом, а правое нижнее горизонтальное отображение мономорфизмом, как следует из леммы 1. Среднее вертикальное отображение является изоморфизмом согласно [8]. Композиция верхних горизонтальных отображений есть первый дифференциал в спектральной последовательности хирургии $d_{1}=1+\Phi$, где $\Phi$ - инволюция на группе $L N_{3}$ (см. [10]). Так как $L_{3}\left(T^{-}\right)$является элементарной 2 -группой, образ отображения $d_{1}$ в верхней строке диаграммы также является элементарной 2 -группой. Групш $L_{3}\left(\widehat{R}_{2}, \mathrm{Id}, 1\right)$ является элементарной 2 -группой. Из рассмотрения правого квадрата диаграммы следует, что образ $d_{1}$ является прямым слагаемым.

Аналогично, рассмотрим коммутативную диаграмму

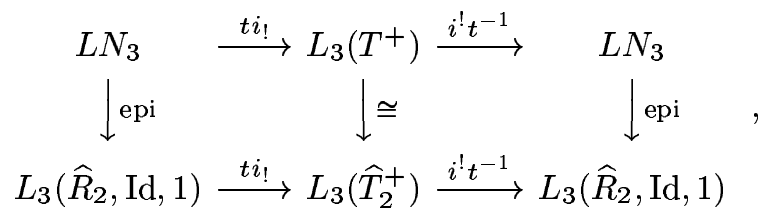


в которой правые горизонтальные отображения имеют изоморфные образы $(\mathbb{Z} / 2)^{2^{r-1}}$ по лемме 1. Композиция верхних горизонтальных отображений есть правьй дифференциал $1-\Phi$ в спектральной последовательности хирургии. Из рассмотрения правого квадрата следует, что образ отображения $1-\Phi$ является элементарной 2-групой и выделяется прямым слагаемым в групе $L N_{3}$. Применение леммы 3 завершает доказательство теоремы 3.

Теперь мы можем вычислить естественные отображения групп Уолла в двустрочной диаграмме (1) для вложений $i: \pi=\mathbb{Z} / 2 \rightarrow D_{r+1}^{ \pm}=G^{ \pm}$. Далее применим эти результаты для вычисления групп $C_{n}\left(D_{r+1}, w\right)$.

Напомним необходимые для вычислений результаты о группах Уолла и группах Браудера-Ливси (см. [8], [9]). Пусть $\Sigma, \Sigma^{\prime}-$ бесконечные абелевы групшы ранга $2^{r-1}-1$, $2^{r-1}+3$ соответственно. Имеют место следуюшие изоморфизмы:

$$
\begin{aligned}
& L_{n}(\pi) \cong \begin{cases}\Sigma \oplus \mathbb{Z}^{2} & \text { при } n=0 \bmod 4, \\
0 & \text { при } n=1 \bmod 4, \\
\Sigma \oplus \mathbb{Z} / 2 & \text { при } n=2 \bmod 4, \\
\mathbb{Z} / 2 & \text { при } n=3 \bmod 4,\end{cases} \\
& L_{n}\left(G^{+}\right) \cong \begin{cases}\Sigma^{\prime} & \text { при } n=0 \bmod 4, \\
(\mathbb{Z} / 2)^{2^{r-1}-r} & \text { при } n=1 \bmod 4, \\
\mathbb{Z} / 2 & \text { при } n=2 \bmod 4, \\
(\mathbb{Z} / 2)^{2^{r-1}+2} & \text { при } n=3 \bmod 4,\end{cases} \\
& L_{n}\left(G^{-}\right) \cong \begin{cases}\mathbb{Z} / 2 & \text { при } n=0 \bmod 4, \\
(\mathbb{Z} / 2)^{2^{r-1}-1} & \text { при } n=1 \bmod 4, \\
\Sigma \oplus \mathbb{Z} / 2 & \text { при } n=2 \bmod 4, \\
(\mathbb{Z} / 2)^{2^{r-1}-r} & \text { при } n=3 \bmod 4 .\end{cases}
\end{aligned}
$$

Группу $L_{n}(\mathbb{Z} \pi, \mathrm{Id}, 1) \cong L N_{n}\left(\pi \rightarrow G^{-}\right) \cong L N_{n+2}\left(\pi \rightarrow G^{+}\right)$обозначим через $L N_{n}$. Тогда $L N_{3} \cong(\mathbb{Z} / 2)^{2^{r}+r-2}$ по теореме 3. Согласно [9] групша $L N_{2}$ изоморфна $\mathbb{Z} / 2$ (Arf-инвариант), и имеет место точная последовательность

$$
0 \longrightarrow L N_{1} \longrightarrow(\mathbb{Z} / 2)^{2^{r-1}+r} \oplus \mathbb{Z}^{2} \longrightarrow L N_{0} \longrightarrow 0 .
$$

Рассмотрим участок двустрочной диаграммы (1)

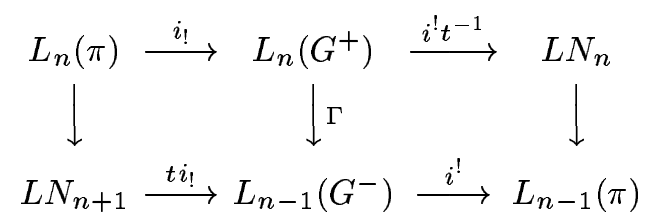

для вложения $i: \pi \rightarrow G^{+}$и аналогичньй участок

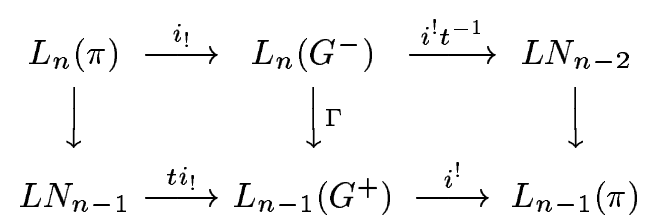

для вложения $i: \pi \rightarrow G^{-}$. 
Tеорема 4. 1) В диаграмме (5) отображсение $i^{!} t^{-1}: L_{n}\left(G^{+}\right) \rightarrow L N_{n}$ мономорфно при $n=1 \bmod 4$, тривиально при $n=2 \bmod 4$, имеет ядро $(\mathbb{Z} / 2)^{2} n p и n=3 \bmod 4$, эпиморфно при $n=0 \bmod 4$.

Отобрахсение $i_{!}: L_{n}(\pi) \rightarrow L_{n}\left(G^{+}\right)$тривиально при $n=1 \bmod 4$, мономорфно при $n=3 \bmod 4$, эпиморфно при $n=2 \bmod 4$, мономорфно скоядром $\mathbb{Z}^{2} \oplus(\mathbb{Z} / 2)^{2^{r-1}-r}$ npu $n=0 \bmod 4$.

2) В диаграмме (6) отображсние $i^{!} t^{-1}: L_{n}\left(G^{-}\right) \rightarrow L N_{n-2}$ мономорфно при $n=1,3 \bmod 4$, тривиально при $n=0 \bmod 4$, эпиморфно при $n=2 \bmod 4$.

Отобрахсение $i_{!}: L_{n}(\pi) \rightarrow L_{n}\left(G^{-}\right)$тривиально при $n=0,1,3 \bmod 4$ и мономорфно с коядром $\mathbb{Z}^{2} \oplus(\mathbb{Z} / 2)^{2^{r-1}-r}$ при $n=2 \bmod 4$.

ДокАЗАТЕЛЬСтво. Рассмотрим только случай 1 ). Отображение $i$ ! эпиморфно в размерности 2, так как оно сохраняет Arf-инвариант. В размерности 1 оно тривиально, так как $L_{1}(\pi)=0$. В этом случае рассмотрим следующий участок диаграммы (1):

$$
\mathbb{Z} / 2 \cong L_{3}(\pi) \stackrel{i_{!}}{\longrightarrow} L_{3}\left(G^{+}\right) \stackrel{i^{!} t^{-1}}{\longrightarrow} L N_{3} \stackrel{t i_{!}}{\longrightarrow} L_{1}\left(G^{-}\right) \longrightarrow 0 .
$$

Гомологии в члене $L_{1}\left(G^{-}\right)$тривиальны, как следует из рассмотрения нижней строки. Гомологии в члене $L N_{3}$ изоморфны гомологиям в члене $\stackrel{i^{!}}{\longrightarrow} L_{2}(\pi) \stackrel{i_{!}}{\longrightarrow}$ нижней строки диаграммы. Рассмотрим коммутативную диаграмму

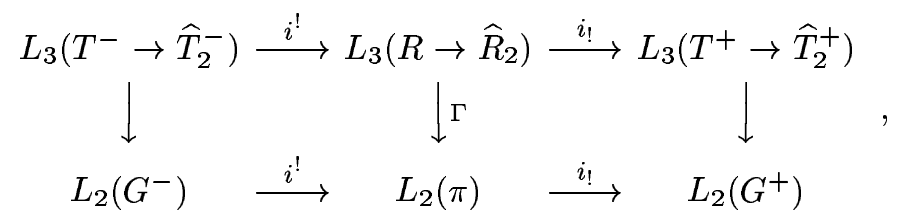

в которой вертикальные отображения являются отображениями из соответствующих относительньх точных последовательностей (3). Гомологии в члене $L_{3}\left(R \rightarrow \widehat{R}_{2}\right)$ равны $(\mathbb{Z} / 2)^{r-1}$. Они изоморфно отображаются в гомологии в члене $L_{2}(\pi)$ нижней строки, так как среднее вертикальное отображение является вложением прямого слагаемого, а правое вертикальное отображение тривиально. Гомологии в члене $L_{3}\left(G^{+}\right)$диаграммы (7) изоморфны прямому слагаемому $\mathbb{Z} / 2$ группы $L_{2}\left(G^{-}\right)$из соответствующей нижней строки, так как в группе $L N_{0}$ Arf-инвариант не реализуется, а отображение $L_{2}\left(G^{-}\right) \stackrel{i^{!}}{\longrightarrow} L_{2}(\pi)$, лежащее в нижней строке, является мономорфизмом на свободной части по лемме 2. Следовательно, в диаграмме (7)

$\operatorname{rang}\left(\operatorname{Im} i^{!} t^{-1}\right)=\operatorname{rang} L N_{3}-\operatorname{rang} L_{1}\left(G^{-}\right)-(r-1)=2^{r}+r-2-\left(2^{r-1}-1\right)-(r-1)=2^{r-1}$.

Отсюда немедленно следуют оба утверждения из 1) в размерности 3. Описание отображений $i^{!} t^{-1}$ в размерностях 1 и 2 не представляет трудностей, так как в диаграмме $(D)$ в этом случае отображение $\mathbb{Z} / 2 \cong L N_{2} \stackrel{t i_{!}}{\rightarrow} L_{0}\left(G^{-}\right) \cong \mathbb{Z} / 2$ является изоморфизмом. Для доказательства утверждений в размерности 0 рассмотрим коммутативную диаграмму

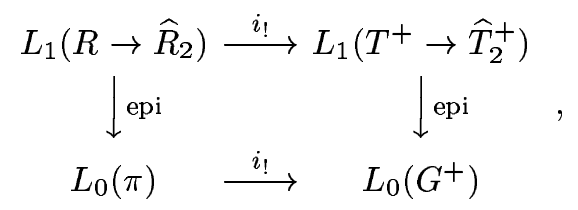


в которой вертикальные отображения лежат в относительных точных последовательностях. Из леммы 2 следует, что верхнее горизонтальное отображение является мономорфизмом. При этом свободная часть переходит в свободную часть с коядром $\mathbb{Z}^{2} \oplus$ $(\mathbb{Z} / 2)^{2^{r-1}-r}$. Так как вертикальные отображения являются эпиморфизмами и нижние групшы не имеют кручения, нижнее горизонтальное отображение имеет такое же

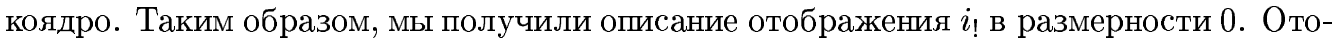
бражение $i ! t^{-1}$ является эпиморфизмом, так как гомологии в члене $L N_{0}$ тривиальны. Это следует из мономорфности отображения $i_{!}: L_{3}(\pi) \rightarrow L_{3}\left(G^{+}\right)$, лежащего в другой строке. Случай 2) рассматривается аналогично. Теорема доказана.

ЗАмечаниЕ. Результаты, полученные в теореме 4, позволяют диаграммньм поиском вычислить почти все отображения и групшы гомологий в двустрочных диаграм$\max (D)$ и $(\widetilde{D})$ для вложений $\mathbb{Z} / 2^{r} \rightarrow D_{r+1}$, рассматриваемых в работе. Однако, в настоящее время автору неизвестны группы гомологий в члене $L_{0}\left(G^{+}\right)$диаграммы $(D)$ и групы гомологий в члене $L_{2}\left(G^{-}\right)$диаграммы $(\widetilde{D})$. Для ответа на этот вопрос достаточно знать отображения в точной последовательности, связьвающей грушы $L N_{0}$ и $L N_{1}$.

4. Реализуемость замкнутыми многообразиями. Согласно результатам [6] (см. также [2], [16]) элементы групшы $L_{n}\left(G^{+}\right)$, не лежашие в ядре отображения $i^{!} t^{-1}$ в диаграмме (5), не реализуются нормальными отображениями замкнутых многообразий. Таким образом, $i^{!} t^{-1}(x)$ при $x \in L_{n}(G)$ является первьм препятствием к реализуемости элемента $x$ нормальньм отображением замкнутых многообразий. Пусть для элемента $x$ первоепрепятствие тривиально, т.е. $i^{!} t^{-1}(x)=0$. Используя гомоморфизм $\Gamma$ в гомологиях из диаграммы (5), можно построить класс $\Gamma(x) \subset L_{n-1}\left(G^{-}\right)$, где отображение $i^{!} t^{-1}$ лежит в диаграмме (6). Согласно [2] этот смежный класс является вторым препятствием к реализуемости; если $0 \notin \Gamma(x)$, то элемент $x$ не реализуется нормальным отображением замкнутых многообразий. Этих двух инвариантов оказывается достаточно для исследования проблемы реализуемости в проективном варианте (см. [2]). Далее этот процесс можно продолжить и получить итерированные инварианты Браудера-Ливси (см. [16]), но для диэдральных 2-групш в рассматриваемом случае нет такой необходимости. Напомним, что $D_{r+1}=G$-группа с тривиальным гомоморфизмом ориентации, а $D_{r+1}^{-}=G^{-}$-группа с гомоморфизмом ориентации $w$, для которого $w(x)=1$, $w(y)=-1$.

ТЕОрема 5. Имеют место следующие изоморфизмы:

$$
C_{n}\left(D_{r+1}\right)=\left\{\begin{array}{ll}
0 & \text { npu } n=1 \bmod 4, \\
\mathbb{Z} / 2 & \text { npu } n=2 \bmod 4, \\
(\mathbb{Z} / 2)^{2} & \text { npu } n=3 \bmod 4,
\end{array} \quad C_{n}\left(D_{r+1}^{-}\right)= \begin{cases}\mathbb{Z} / 2 & \text { npu } n=0 \bmod 4, \\
0 & \text { npu } n=1,3 \bmod 4 .\end{cases}\right.
$$

ДокАЗАТЕЛЬСтво. Всенеобходимые отображения вычислены в теореме 4. Рассмотрим сначала случай нетривиальной ориентации. Отображения $i^{!} t^{-1}$ в диаграмме (6) являются мономорфизмами в нечетной размерности. Следовательно, все элементы групा $L_{2 n+1}\left(G^{-}\right)$не реализуются нормальными отображениями замкнутых многообразий. Группа $L_{0}\left(G^{-}\right)$изоморфна $\mathbb{Z} / 2$ и согласно [2] образ групшы $C_{0}\left(D_{r+1}^{-}\right)$в проективной группе $L^{p}$ равен $\mathbb{Z} / 2$. Отсюда следует утверждение теоремы в случае нетривиальной ориентации. 
В случае тривиальной ориентации отображение $i^{!} t^{-1}$ в диаграмме (5) мономорфно в размерности 1 , а в размерности 3 имеет ядро $(\mathbb{Z} / 2)^{2}$. Согласно [2] образ группы $C_{3}\left(D_{r+1}\right)$ в проективной группе равен $(\mathbb{Z} / 2)^{2}$. Таким образом, в размерности 3 верхняя и нижняя оценки групшы $C_{3}\left(D_{r+1}\right)$ совпадают и, следовательно, $C_{3}\left(D_{r+1}\right) \cong(\mathbb{Z} / 2)^{2}$. В размерности 2 повторяем рассуждения, относящиеся к размерности 0 неориентируемого случая. Теорема доказана.

В размерности 0 в ориентируемом случае из диаграмм (5) и (6) также удается получить верхнюю оценку $\mathbb{Z}^{r+1}$ на группу $C_{0}\left(D_{r+1}\right)$, но она значительно превосходит нижнюю оценку $\mathbb{Z}$ из работы [2]. В размерности 2 в неориентируемом случае ситуация аналогична.

\section{СПИСОК ЦИТИРОВАННОЙ ЛИТЕРАТУРЫ}

[1] Wall C. T. C. Surgery on Compact Manifolds. London: Acad. Press, 1970.

[2] Hambleton I. Projective surgery obstructions // Lecture Notes in Math. 1982. V. 967. P. 101-131.

[3] Hambleton I., Milgram R. J., Taylor L., Williams B. Surgery with finite fundamental group // Proc. London Math. Soc. (3). 1988. V. 56. P. 349-379.

[4] Morgan R. J. Surgery with finite fundamental group. II. The oozing conjecture // Pacific J. Math. 1991. V. 151. P. 117-149.

[5] Browder W., Livesay R. Fixed point free involutions of homotopy spheres // Tôhoku J. Math. (2). 1973. V. 25. P. 69-88.

[6] Cappell S. E., Shaneson J. L. Pseudo-free actions // Lecture Notes in Math. 1979. V. 763. P. 395-447.

[7] Ranicki A. The $L$-theory of twisted quadratic extensions // Canad. J. Math. 1987. V. 39. P. 345-364.

[8] Wall C. T. C. On the classification of Hermitian forms. VI. Group rings // Ann. of Math. (2). 1976. V. 103. P. $1-80$.

[9] Муранов Ю.В.Когомологии Тейта и группы Браудера-Ливси диэдральных групп // Матем. заметки. 1993. Т. 54. № 2. С. 44-55.

[10] Хэмблтон И., Харшиладзе А. Ф. Спектральная последовательность в теории перестроек // Матем. сб. 1992. Т. 183. № 9. С. 3-14.

[11] Муранов Ю. В. Относительные группы Уолла и декорации // Матем. сб. 1994. Т. 185. № 12. C. $79-100$.

[12] Муранов Ю. В. K-группы квадратичных расширений колец // Матем. заметки. 1995. T. 58. № 2. C. $272-280$.

[13] Cavicchioli A., Muranov Y.V., Repovs D. Spectral Sequences in $K$-Theory for a Twisted Quadratic Extension. Preprint. V. 34: University of Ljubljana, 1996.

[14] Hambleton I., Ranicki A., Taylor L. Round L-theory // J. Pure Appl. Algebra. 1987. V. 47. P. 131-134.

[15] Hambleton I., Taylor L., Williams B. An introduction to maps between surgery obstruction groups // Lecture Notes in Math. 1984. V. 1051. P. 49-127.

[16] Харшиладзе А. Ф. Перестройка многообразий с конечными фундаментальными группами // УМH. 1987. T. 42. № 4. C. 55-85.

Владимирский государственный университет

University of Ljubljana (Slovenija), Institute of Mathematics, Physics and Mechanics

Поступило E-mail: muranov@vpti.vladimir.su, dusan.repovs@uni-lj.si 